Research Article

\title{
Some Density Results on Sets of Primes for Hecke Eigenvalues
}

\author{
Aiyue Zou, Huixue Lao $(D$, and Shu Luo \\ School of Mathematics and Statistics, Shandong Normal University, Jinan, Shandong 250358, China \\ Correspondence should be addressed to Huixue Lao; lhxsdnu@163.com
}

Received 3 June 2021; Accepted 26 June 2021; Published 22 July 2021

Academic Editor: Jie Wu

Copyright (c) 2021 Aiyue Zou et al. This is an open access article distributed under the Creative Commons Attribution License, which permits unrestricted use, distribution, and reproduction in any medium, provided the original work is properly cited.

Let $f$ and $g$ be two distinct holomorphic cusp forms for $S L_{2}(\mathbb{Z})$, and we write $\lambda_{f}(n)$ and $\lambda_{g}(n)$ for their corresponding Hecke eigenvalues. Firstly, we study the behavior of the signs of the sequences $\left\{\lambda_{f}(p) \lambda_{f}\left(p^{j}\right)\right\}$ for any even positive integer $j$. Moreover, we obtain the analytic density for the set of primes where the product $\lambda_{f}\left(p^{i}\right) \lambda_{f}\left(p^{j}\right)$ is strictly less than $\lambda_{g}\left(p^{i}\right) \lambda_{g}\left(p^{j}\right)$. Finally, we investigate the distribution of linear combinations of $\lambda_{f}\left(p^{j}\right)$ and $\lambda_{g}\left(p^{j}\right)$ in a given interval. These results generalize previous ones.

\section{Introduction}

Let $H_{k}^{*}$ be the set of all normalized Hecke primitive cusp forms of even integral weight $k \geq 2$ for the full modular group $S L_{2}(\mathbb{Z})$ denoted by $\lambda_{f}(n)$ the $n$-th Hecke eigenvalue of $f \in H_{k}^{*}$. The Hecke eigenvalues of cusp forms have been extensively studied (see, e.g., [1-7]). From the theory of Hecke operators, we know that $\lambda_{f}(n)$ satisfies the standard Hecke relation as follows: for any integers $m, n \geq 1$,

$$
\lambda_{f}(m) \lambda_{f}(n)=\sum_{d \mid(m, n)} \lambda_{f}\left(\frac{m n}{d^{2}}\right) .
$$
and

In particular, $\lambda_{f}(n)$ is a real and multiplicative function

$$
\lambda_{f}(1)=1 .
$$

Furthermore, it is also known that $\lambda_{f}(n)$ satisfies the Ramanujan conjecture [8]:

$$
\left|\lambda_{f}(n)\right| \leq d(n),
$$

where $d(n)$ is the Dirichlet divisor function.

There are many papers that focus on the sign changes of the Hecke eigenvalues $\lambda_{f}(n)$. It is well known that $\left\{\lambda_{f}(n)\right\}_{n>1}$ changes sign infinitely often. Meher et al. [9] studied the distribution of the signs of $\left\{\lambda_{f}\left(p^{j}\right)\right\}$ as $p$ varies over the prime numbers. They calculated the natural density of the sets explicitly in terms of the celebrated Sato-Tate conjecture (see Theorem B in [10]). In [11], a joint version of the pair-Sato-Tate conjecture (as outlined in Proposition 2.2 in [12]) gives the result that the set $\left\{p \mid \lambda_{f}\left(p^{j}\right) \lambda_{g}\left(p^{j}\right)<0\right\}$ has natural density $1 / 2$ for any odd positive integer $j$.

In this paper, based on the now-proven Sato-Tate conjecture, we first study the behavior of the signs of $\left\{\lambda_{f}(p) \lambda_{f}\left(p^{j}\right)\right\}$ for any even positive integer $j$.

Theorem 1. Let $f \in H_{k}^{*}$ be a cusp form. Then, for any even positive integer $j$, the sets $\left\{p \mid \lambda_{f}(p) \lambda_{f}\left(p^{j}\right)>0\right\}$ and $\left\{p \mid \lambda_{f}(p) \lambda_{f}\left(p^{j}\right)<0\right\}$ both have natural density $1 / 2$.

In [13], Kowalski et al. first proved that if the signs of $\lambda_{f}(p)$ and $\lambda_{g}(p)$ coincide for all primes up to the exceptional set of analytic density at most $1 / 32$, then $f=g$. Subsequently, Matomäki [14] improved the above result by utilizing linear programming to take full advantage of all the available information.

Inspired by [13], Chiriac [15] started to compare Hecke eigenvalues over prime numbers and simultaneously showed that the sets of primes for $\lambda_{f}(p)<\lambda_{g}(p)$ and $\lambda_{f}^{2}(p)<\lambda_{g}^{2}(p)$ both have analytic density at least $1 / 16$. Notice that the pairSato-Tate conjecture yields a stronger result for the former set in [15] with natural density $1 / 2$ in replace of at least $1 / 16$ (see Proposition 2.1 (iii) in [16]). Of course, this result is also valid for the analytic density since the existence of the natural density implies that of the analytic density, and they are equal. 
Most recently, Lao [17] further studied Chiriac's questions [15] by considering the Hecke eigenvalues at prime powers. She established that the sets $\left\{p \mid \lambda_{f}\left(p^{j}\right)<\lambda_{g}\left(p^{j}\right)\right\}$ with $1 \leq j \leq 8$ and $\left\{p \mid \lambda_{f}^{2}\left(p^{j}\right)<\lambda_{g}^{2}\left(p^{j}\right)\right\} \quad$ with $1 \leq j \leq 4$ have analytic density at least $\left(1 / 16[(j+1) / 2]^{2}\right)$ and $\left(1 / 4 j(j+1)^{2}\right)$, respectively.

In this paper, our second aim is to obtain the analytic density for the set $\left\{p \mid \lambda_{f}\left(p^{i}\right) \lambda_{f}\left(p^{j}\right)<\lambda_{g}\left(p^{i}\right) \lambda_{g}\left(p^{j}\right)\right\}$ with $j \geq 1$ and $0 \leq i \leq j$.

Theorem 2. Let $f, g \in H_{k}^{*}$ be two distinct cusp forms. Then, for $j \geq 1$ and $0 \leq i \leq j$, the set $\left\{p \mid \lambda_{f}\left(p^{i}\right) \lambda_{f}\left(p^{j}\right)<\lambda_{g}\right.$ $\left.\left(p^{i}\right) \lambda_{g}\left(p^{j}\right)\right\}$ has analytic density at least $\left(\left(1+i-\delta_{1}\right) /\right.$ $\left.4\left(i j+j+\delta_{2}\right)^{2}\right)$, where

$$
\begin{aligned}
& \delta_{1}= \begin{cases}0, & \text { if } i \neq j, \\
1, & \text { if } i=j,\end{cases} \\
& \delta_{2}= \begin{cases}i+1, & \text { if } 2+i+j, \\
0, & \text { if } 2 \mid i+j .\end{cases}
\end{aligned}
$$

Remark 1. By symmetry, for $j \geq 1$ and $0 \leq i \leq j$, Theorem 2 implies that the set $\left\{p \mid \lambda_{f}\left(p^{i}\right) \lambda_{f}\left(p^{j}\right)>\lambda_{g}\left(p^{i}\right) \lambda_{g}\left(p^{j}\right)\right\}$ has analytic density at least $\left(\left(1+i-\delta_{1}\right) / 4\left(i j+j+\delta_{2}\right)^{2}\right)$. Thus, for $j \geq 1$ and $0 \leq i \leq j$, the set $\left\{p \mid \lambda_{f}\left(p^{i}\right) \lambda_{f}\right.$ $\left.\left(p^{j}\right)=\lambda_{g}\left(p^{i}\right) \lambda_{g}\left(p^{j}\right)\right\}$ has analytic density at most $1-\left(\left(1+i-\delta_{1}\right) / 2\left(i j+j+\delta_{2}\right)^{2}\right)$.
Remark 2. One can easily find that for $i=0, j=1$, or $i=j=1$, we have $\left(\left(1+i-\delta_{1}\right) / 4\left(i j+j+\delta_{2}\right)^{2}\right)=(1 / 16)$, which are consistent with the numerical values of Chiriac's results (see Theorems 1 and 1.3 in [15]). By comparison, $\left(\left(1+i-\delta_{1}\right) / 4\left(i j+j+\delta_{2}\right)^{2}\right)=\left(1 / 16[(j+1) / 2]^{2}\right)$ with $i=0,1 \leq j \leq 8$, and $\left(\left(1+i-\delta_{1}\right) / 4\left(i j+j+\delta_{2}\right)^{2}\right)=(1 / 4 j$ $\left.(j+1)^{2}\right)$ with $1 \leq i=j \leq 4$. Hence, Theorem 2 also generalizes previous results of Lao (see Theorems 1 and 2 in [17]).

It is natural to ask whether Theorem 2 can be refined by the pair-Sato-Tate conjecture. In fact, with the help of this conjecture, some specific cases can be dealt with by calculating the corresponding double integral.

Finally, we concern the distribution of linear combinations of $\lambda_{f}\left(p^{j}\right)$ and $\lambda_{g}\left(p^{j}\right)$ in a specified interval. Chiriac and Jorza (see Proposition 5.9 in [18]) obtained the density bound for the set $\left\{v \mid a<c_{1} a_{v}\left(\pi_{1}\right)+c_{2} a_{v}\left(\pi_{2}\right)<b\right\}$ in the context of unitary cuspidal representations $\pi$ that satisfy the Ramanujan conjecture. For holomorphic cusp forms, we establish the following theorem.

Theorem 3. Let $f, g \in H_{k}^{*}$ be two distinct cusp forms. Let $a, b, c_{1}, c_{2} \in \mathbb{R}$ with $a<b$. Then, for $j \geq 1$, the set $\left\{p \mid a<c_{1} \lambda_{f}\left(p^{j}\right)+c_{2} \lambda_{g}\left(p^{j}\right)<b\right\}$ has analytic density at least

$$
\begin{aligned}
& \frac{(j+1)\left(c_{1}^{4}+c_{2}^{4}\right)+\delta_{3}\left(c_{1}^{3}+c_{2}^{3}\right)+6 c_{1}^{2} c_{2}^{2}+\left((a+b)^{2}+2 a b\right)\left(c_{1}^{2}+c_{2}^{2}\right)+a^{2} b^{2}}{2\left((j+1)^{2}\left(\left|c_{1}\right|+\left|c_{2}\right|\right)^{2}+(j+1)(|a|+|b|)\left(\left|c_{1}\right|+\left|c_{2}\right|\right)+|a b|\right)^{2}} \\
& -\frac{c_{1}^{2}+c_{2}^{2}+a b}{2\left((j+1)^{2}\left(\left|c_{1}\right|+\left|c_{2}\right|\right)^{2}+(j+1)(|a|+|b|)\left(\left|c_{1}\right|+\left|c_{2}\right|\right)+|a b|\right)},
\end{aligned}
$$

where

$$
\delta_{3}= \begin{cases}0, & \text { if } j \text { is odd } \\ 1, & \text { if } j \text { is even }\end{cases}
$$

The proofs of Theorems 2 and 3 rely on Lemma 4 involving the analytic density of a particular set of primes. Recently, there was a big breakthrough on the automorphy of all symmetric powers for cuspidal Hecke eigenforms (Theorem A in [19]), which implies that the $L$-function $L\left(\operatorname{sym}^{j} f, s\right)$ is automorphic for $j \geq 1$ and $f \in H_{k}^{*}$. Then, with the help of the properties of symmetric power $L$-functions and their Rankin-Selberg $L$-functions, we obtain the desired results.

\section{Preliminaries}

2.1. Primitive Automorphic L-Functions. In this section, we will briefly recall some fundamental facts about primitive automorphic $L$-functions and give the main tools and definitions. For more details to learn automorphic $L$-functions, refer [17, 20-28].

Let $f, g \in H_{k}^{*}$ be two cusp forms. The $j$-th symmetric power $L$-function attached to $f$ is defined by

$$
L\left(\operatorname{sym}^{j} f, s\right)=\prod_{p} \prod_{m=0}^{j}\left(1-\frac{\alpha_{f}(p)^{j-m} \beta_{f}(p)^{m}}{p^{s}}\right)^{-1}, \quad \operatorname{Re}(s)>1,
$$

where $\alpha_{f}(p)$ and $\beta_{f}(p)$ are two complex numbers with 


$$
\begin{aligned}
\lambda_{f}(p) & =\alpha_{f}(p)+\beta_{f}(p) \\
\left|\alpha_{f}(p)\right| & =\left|\beta_{f}(p)\right|=\alpha_{f}(p) \beta_{f}(p)=1 .
\end{aligned}
$$

One can write it as a Dirichlet series: for $\operatorname{Re}(s)>1$,

$$
L\left(\operatorname{sym}^{j} f, s\right)=\sum_{n=1}^{\infty} \frac{\lambda_{\mathrm{sym}^{j} f}(n)}{n^{s}}=\prod_{p}\left(1+\sum_{v \geq 1} \frac{\lambda_{\mathrm{sym}^{j} f}\left(p^{v}\right)}{p^{\nu s}}\right),
$$

where $\lambda_{\mathrm{sym}^{j} f}(n)$ is a real multiplicative function, and

$$
\lambda_{\text {sym }^{j} f}(p)=\sum_{m=0}^{j} \alpha_{f}(p)^{j-2 m}=\lambda_{f}\left(p^{j}\right) .
$$

The Rankin-Selberg $L$-function attached to $\operatorname{sym}^{i} f$ and sym $^{j} \mathrm{~g}$ is defined by

$$
\begin{aligned}
L\left(\operatorname{sym}^{i} f \times \operatorname{sym}^{j} g, s\right) & =\prod_{p} \prod_{m=0}^{i} \prod_{m^{\prime}=0}^{j}\left(1-\frac{\alpha_{f}(p)^{i-2 m} \alpha_{g}(p)^{j-2 m^{\prime}}}{p^{s}}\right)^{-1} \\
& =\sum_{n=1}^{\infty} \frac{\lambda_{\text {sym }^{i} f \times \operatorname{sym}^{j} g}(n)}{n^{s}}, \quad \operatorname{Re}(s)>1,
\end{aligned}
$$

where $\lambda_{\text {sym }^{i} f \times \operatorname{sym}^{j} g}(n)$ is a real multiplicative function, and

$$
\begin{aligned}
\lambda_{\text {sym }^{i} f \times \operatorname{sym}^{j} g}(p) & =\sum_{m=0}^{i} \sum_{m^{\prime}=0}^{j} \alpha_{f}(p)^{i-2 m} \alpha_{g}(p)^{j-2 m^{\prime}} \\
& =\lambda_{\text {sym }^{i} f}(p) \lambda_{\text {sym }^{j} g}(p) .
\end{aligned}
$$

We make the convention that

$$
\left\{\begin{array}{l}
L\left(\operatorname{sym}^{0} f, s\right)=\zeta(s), \\
L\left(\operatorname{sym}^{1} f, s\right)=L(f, s), \\
L\left(\operatorname{sym}^{0} f \times \operatorname{sym}^{j} f, s\right)=L\left(\operatorname{sym}^{j} f, s\right) .
\end{array}\right.
$$

A key ingredient of proving Theorems 2 and 3 is the analytic properties of various automorphic $L$-functions. By a series of deep works [29-36], we learn that for $1 \leq j \leq 8$, $L\left(\operatorname{sym}^{j} f, s\right)$ is an automorphic $L$-function. Recently, Newton and Thorne (see Theorem A in [19]) proved the automorphy of the symmetric power lifting $\operatorname{sym}^{j} f$ for $j \geq 1$ and $f \in H_{k}^{*}$. Hence, by standard arguments, we have the following.

Lemma 1. Let $f \in H_{k}^{*}$ be a cusp form and $L\left(\operatorname{sym}^{j} f, s\right)$ be defined as in (8). For $j \geq 1, L\left(\operatorname{sym}^{j} f, s\right)$ has an analytic continuation as an entire function in the whole complex plane $\mathbb{C}$.

Combining Lemma 1 with (10), we deduce that for $j \geq 1$,

$$
\sum_{p} \frac{\lambda_{\mathrm{sym}^{j} f}(p)}{p^{s}}=O(1), \quad \text { as } s \longrightarrow 1^{+} \text {. }
$$

Moreover, based on the automorphy of $\operatorname{sym}^{j} f$ for $j \geq 1$ and the work [37-42] on the Rankin-Selberg theory, we have the following.

Lemma 2. Let $f, g \in H_{k}^{*}$ be two cusp forms and $L\left(s^{\prime} m^{i} f \times\right.$ $\left.\operatorname{sym}^{j} g, s\right)$ be defined as in (12). For $i, j \geq 1$, $L\left(\operatorname{sym}^{i} f \times \operatorname{sym}^{j} g, s\right)$ has an analytic continuation as an entire function in the whole complex plane $\mathbb{C}$ (except possibly for simple poles at $s=0,1$ when $\operatorname{sym}^{j} f \cong \operatorname{sym}^{j} \mathrm{~g}$ ).

Thus, when $f=g, s=1$ is a simple pole of $L\left(\operatorname{sym}^{j} f \times \operatorname{sym}^{j} g, s\right)$. By (12), we have

$$
\sum_{p} \frac{\lambda_{\text {sym }^{j} f \times \operatorname{sym}^{j} g}(p)}{p^{s}}=\sum_{p} \frac{1}{p^{s}}+O(1), \quad \text { as } s \longrightarrow 1^{+} .
$$

In other cases, $L\left(\operatorname{sym}^{i} f \times \operatorname{sym}^{j} g, s\right)$ is an entire function and does not vanish at $s=1$. Thus,

$$
\sum_{p} \frac{\lambda_{\text {sym }^{i} f \times \text { sym }^{j}}(p)}{p^{s}}=O(1), \quad \text { as } s \longrightarrow 1^{+} \text {. }
$$

2.2. Sato-Tate Conjecture. Firstly, let us introduce the definition of natural density.

Definition 1. For a subset $\mathscr{A} \subseteq \mathbb{P}$ which denotes the set of all primes, the natural density of $\mathscr{A}$ in $\mathbb{P}$ is defined as

$$
\lim _{x \rightarrow \infty} \frac{\#\{p \leq x \mid p \in \mathscr{A}\}}{\#\{p \leq x \mid p \in \mathbb{P}\}},
$$

provided the limit exists.

Secondly, let us define the Sato-Tate measure and state the Sato-Tate conjecture (see Theorem 2.3 in [9]), which will be used to prove Theorem 1.

Definition 2. The Sato-Tate measure $\mu_{\mathrm{ST}}$ is the probability measure on $[0, \pi]$ given by $(2 / \pi) \sin ^{2} \theta \mathrm{d} \theta$.

For any subinterval $I \subseteq[0, \pi]$, one has

$$
\pi_{I}(x)=\#\left\{p \leq x \mid \theta_{p} \in I\right\} .
$$

Lemma 3. Let $f \in H_{k}^{*}$ be a cusp form. The sequence $\left\{\theta_{p}\right\}$ is equidistributed in $[0, \pi]$ with respect to the Sato-Tate measure $\mu_{S T}$. In particular, for any subinterval $I \subseteq[0, \pi]$, we have

$$
\lim _{x \longrightarrow \infty} \frac{\pi_{I}(x)}{\pi(x)}=\mu_{\mathrm{ST}}(I)=\frac{2}{\pi} \int_{I} \sin ^{2} \theta \mathrm{d} \theta .
$$

Lemma 3 implies that if $\mathscr{A}$ is a finite set, then the natural density of the set $\left\{p \mid \theta_{p} \in \mathscr{A}\right\}$ is 0 . 
2.3. An Analytic Density Lemma. We also recall the definition of analytic density.

Definition 3. A set $\mathscr{B}$ of primes is said to have analytic density (or Dirichlet density) $\delta>0$ if and only if,

$$
\sum_{p \in \mathscr{B}} \frac{1}{p^{s}} \sim \delta \sum_{p} \frac{1}{p^{s}}, \quad \text { as } s \longrightarrow 1^{+} \text {. }
$$

In order to prove Theorems 2 and 3, we need the following lemma, which is inspired by the ideas outlined in Section 3 of [5].

Lemma 4. Let $h_{p}$ be real numbers only determined by prime $p$ and satisfy $\left|h_{p}\right| \leq B$ with a bound $B$ that does not depend on $p$. There exist real constants $m$ and $M$ such that as $s \longrightarrow 1^{+}$, the following equalities hold:

$$
\begin{aligned}
& \sum_{p} \frac{h_{p}^{2}}{p^{s}}=m \sum_{p} \frac{1}{p^{s}}+O(1), \\
& \sum_{p} \frac{h_{p}}{p^{s}}=M \sum_{p} \frac{1}{p^{s}}+O(1) .
\end{aligned}
$$

Then, the set $\mathscr{B}=\left\{p \mid h_{p}<0\right\}$ has analytic density at least $\left((m-M B) / 2 B^{2}\right)$.

Proof. If $p \notin \mathscr{B}, h_{p} \geq 0$, and therefore $h_{p}^{2} \leq B h_{p}$, whereas if $p \in \mathscr{B}$, then $-h_{p} \leq B$ and $-B h_{p} \leq B^{2}$. We see that

$$
\begin{aligned}
\sum_{p} \frac{h_{p}^{2}}{p^{s}} & =\sum_{p \in \mathscr{B}} \frac{h_{p}^{2}}{p^{s}}+\sum_{p \notin \mathscr{B}} \frac{h_{p}^{2}}{p^{s}} \\
& \leq B^{2} \sum_{p \in \mathscr{B}} \frac{1}{p^{s}}+B\left(\sum_{p} \frac{h_{p}}{p^{s}}-\sum_{p \in \mathscr{B}} \frac{h_{p}}{p^{s}}\right)+O(1) \\
& \leq 2 B^{2} \sum_{p \in \mathscr{B}} \frac{1}{p^{s}}+B \sum_{p} \frac{h_{p}}{p^{s}}+O(1), \quad \text { as } s \longrightarrow 1^{+} .
\end{aligned}
$$

Inserting (23) into (24), we get

$$
\sum_{p} \frac{h_{p}^{2}}{p^{s}} \leq 2 B^{2} \sum_{p \in \mathscr{B}} \frac{1}{p^{s}}+M B \sum_{p} \frac{1}{p^{s}}+O(1), \quad \text { as } s \longrightarrow 1^{+} \text {. }
$$

Comparing (22) with (25) leads to

$$
\frac{m-M B}{2 B^{2}} \sum_{p} \frac{1}{p^{s}}+O(1) \leq \sum_{p \in \mathscr{B}} \frac{1}{p^{s}}, \quad \text { as } s \longrightarrow 1^{+},
$$

i.e., the set $\mathscr{B}$ has analytic density at least $\left((m-M B) / 2 B^{2}\right)$.

\section{Proof of Theorem 1}

By (9), for any prime $p$, we can write,

$$
\lambda_{f}(p)=2 \cos \theta_{p}
$$

for some $\theta_{p} \in[0, \pi]$. And, $\lambda_{f}\left(p^{j}\right)$ is expressible by the following elementary trigonometric formula:

$$
\lambda_{f}\left(p^{j}\right)=\frac{\sin \left((j+1) \theta_{p}\right)}{\sin \theta_{p}}
$$

When the values of $\theta_{p}$ are 0 or $\pi$, the values of $\lambda_{f}\left(p^{j}\right)$ are $j+1$ or $(-1)^{j}(j+1)$.

Then, we have

$$
\begin{aligned}
\lambda_{f}(p) \lambda_{f}\left(p^{j}\right) & =\frac{\sin \left(2 \theta_{p}\right)}{\sin \theta_{p}} \cdot \frac{\sin \left((j+1) \theta_{p}\right)}{\sin \theta_{p}} \\
& =2 \cos \theta_{p} \cdot \frac{\sin \left((j+1) \theta_{p}\right)}{\sin \theta_{p}}, \quad \theta_{p} \in(0, \pi) .
\end{aligned}
$$

Since the set $\left\{p \mid \theta_{p}=0\right.$ or $\left.\pi\right\}$ has natural density 0 which only has finitely many primes, we may assume that $\theta_{p} \in(0, \pi)$. For $\theta_{p} \in(0, \pi)$, we know that $\sin \theta_{p}>0$. Hence, the sign of $\lambda_{f}(p) \lambda_{f}\left(p^{j}\right)$ is the same as the sign of $\cos \theta_{p} \sin \left((j+1) \theta_{p}\right)$.

The proof of Theorem 1 can be divided into two cases when $j \equiv 2(\bmod 4)$ and $j \equiv 0(\bmod 4)$.

3.1. $j \equiv 2(\bmod 4)$. Assume that $j \equiv 2(\bmod 4)$, we get

$$
j=4\left[\frac{j}{4}\right]+2
$$

Then, we observe that 


$$
\begin{aligned}
& \cos \theta_{p} \sin \left((j+1) \theta_{p}\right)>0 \Longleftrightarrow \\
& \theta_{p} \in \bigcup_{m=1}^{[j / 4]+1}\left(\frac{(2 m-2) \pi}{j+1}, \frac{(2 m-1) \pi}{j+1}\right) \cup\left(\frac{\pi}{2}, \frac{(j+2) \pi}{2(j+1)}\right) \bigcup_{m=[j / 4]+2}^{2[j / 4]+1}\left(\frac{(2 m-1) \pi}{j+1}, \frac{2 m \pi}{j+1}\right), \\
& \cos \theta_{p} \sin \left((j+1) \theta_{p}\right)<0 \Longleftrightarrow \\
& \theta_{p} \in \bigcup_{m=1}^{[j / 4]}\left(\frac{(2 m-1) \pi}{j+1}, \frac{2 m \pi}{j+1}\right) \bigcup\left(\frac{j \pi}{2(j+1)}, \frac{\pi}{2}\right) \bigcup_{m=[j / 4]+1}^{2[j / 4]+1}\left(\frac{2 m \pi}{j+1}, \frac{(2 m+1) \pi}{j+1}\right) .
\end{aligned}
$$

Especially, we rewrite the third term of the right-hand side of (31) and (32) by using (30).

$$
\begin{aligned}
\bigcup_{m=[j / 4]+2}^{2[j / 4]+1}\left(\frac{(2 m-1) \pi}{j+1}, \frac{2 m \pi}{j+1}\right) & =\bigcup_{m=1}^{[j / 4]}\left(\frac{(4[j / 4]-2 m+3) \pi}{j+1}, \frac{(4[j / 4]-2 m+4) \pi}{j+1}\right) \\
& =\bigcup_{m=1}^{[j / 4]}\left(\frac{(j-2 m+1) \pi}{j+1}, \frac{(j-2 m+2) \pi}{j+1}\right), \\
\bigcup_{m=[j / 4]+1}^{2[j / 4]+1}\left(\frac{2 m \pi}{j+1}, \frac{(2 m+1) \pi}{j+1}\right) & =\bigcup_{m=1}^{[j / 4]+1}\left(\frac{(4[j / 4]-2 m+4) \pi}{j+1}, \frac{(4[j / 4]-2 m+5) \pi}{j+1}\right) \\
& =\bigcup_{m=1}^{[j / 4]+1}\left(\frac{(j-2 m+2) \pi}{j+1}, \frac{(j-2 m+3) \pi}{j+1}\right) .
\end{aligned}
$$

Next, we consider the sets $A$ and $B$ consisting of the following forms:

$$
\begin{aligned}
A & =\bigcup_{m=1}^{[j / 4]+1}\left(\frac{(2 m-2) \pi}{j+1}, \frac{(2 m-1) \pi}{j+1}\right) \cup\left(\frac{\pi}{2}, \frac{(j+2) \pi}{2(j+1)}\right) \bigcup_{m=1}^{[j / 4]}\left(\frac{(j-2 m+1) \pi}{j+1}, \frac{(j-2 m+2) \pi}{j+1}\right) \\
& =\bigcup_{m=1}^{[j / 4]+1} I_{m} \cup J \bigcup_{m=1}^{[j / 4]} K_{m}, \\
B & =\bigcup_{m=1}^{[j / 4]}\left(\frac{(2 m-1) \pi}{j+1}, \frac{2 m \pi}{j+1}\right) \bigcup\left(\frac{j \pi}{2(j+1)}, \frac{\pi}{2}\right) \bigcup_{m=1}^{[j / 4]+1}\left(\frac{(j-2 m+2) \pi}{j+1}, \frac{(j-2 m+3) \pi}{j+1}\right) \\
& =\bigcup_{m=1}^{[j / 4]} K_{m}^{\prime} \bigcup J^{\prime} \bigcup_{m=1}^{[j / 4]+1} I_{m}^{\prime} .
\end{aligned}
$$

We will prove the results in Theorem 1 by showing that the Sato-Tate measure of the two sets $A$ and $B$ is equal, i.e., $\mu_{\mathrm{ST}}(A)=\mu_{\mathrm{ST}}(B)$. Since the sets $A$ and $B$ are divided into disjoint intervals given in (34), it is sufficient to prove that $\mu_{\mathrm{ST}}\left(I_{m}\right)=\mu_{\mathrm{ST}}\left(I_{m}^{\prime}\right)$ for each $m$ with $1 \leq m \leq[j / 4]+1$ and $\mu_{\mathrm{ST}}\left(K_{m}\right)=\mu_{\mathrm{ST}}\left(K_{m}^{\prime}\right)$ for each $m$ with $1 \leq m \leq[j / 4]$ and $\mu_{\mathrm{ST}}(J)=\mu_{\mathrm{ST}}\left(J^{\prime}\right)$.
Now, we restrict our attention to prove $\mu_{\mathrm{ST}}(J)=\mu_{\mathrm{ST}}\left(J^{\prime}\right)$. Using the fact

$$
\int \sin ^{2} x \mathrm{~d} x=\frac{x}{2}-\frac{\sin 2 x}{4}+C
$$

we obtain 


$$
\int_{J} \sin ^{2} x \mathrm{~d} x=\frac{\pi}{4(j+1)}-\frac{1}{4} \sin \left(\frac{(j+2) \pi}{j+1}\right) .
$$

On the other hand, we have

$$
\int_{J^{\prime}} \sin ^{2} x \mathrm{~d} x=\frac{\pi}{4(j+1)}+\frac{1}{4} \sin \left(\frac{j \pi}{j+1}\right) .
$$

We know that

$$
\sin \left(\frac{(j+2) \pi}{j+1}\right)=\sin \left(\pi+\frac{\pi}{j+1}\right)=-\sin \left(\frac{j \pi}{j+1}\right) .
$$

Thus, the second term on the right-hand side of equations (36) and (37) is same. Therefore, we see that

$$
\int_{J} \sin ^{2} x \mathrm{~d} x=\int_{J^{\prime}} \sin ^{2} x \mathrm{~d} x \text {. }
$$

Let us prove $\mu_{\mathrm{ST}}\left(K_{m}\right)=\mu_{\mathrm{ST}}\left(K_{m}^{\prime}\right)$. Applying (35) again yields the following equations:

$$
\begin{aligned}
& \int_{K_{m}} \sin ^{2} x \mathrm{~d} x=\frac{\pi}{2(j+1)}-\frac{1}{4}\left(\sin \left(\frac{2(j-2 m+2) \pi}{j+1}\right)-\sin \left(\frac{2(j-2 m+1) \pi}{j+1}\right)\right), \\
& \int_{K_{m}^{\prime}} \sin ^{2} x \mathrm{~d} x=\frac{\pi}{2(j+1)}-\frac{1}{4}\left(\sin \left(\frac{4 m \pi}{j+1}\right)-\sin \left(\frac{2(2 m-1) \pi}{j+1}\right)\right) .
\end{aligned}
$$

It is easy to find that

$$
\begin{aligned}
\sin \left(\frac{2(j-2 m+2) \pi}{j+1}\right) & =\sin \left(2 \pi+\frac{2(1-2 m) \pi}{j+1}\right) \\
& =-\sin \left(\frac{2(2 m-1) \pi}{j+1}\right), \\
\sin \left(\frac{2(j-2 m+1) \pi}{j+1}\right) & =\sin \left(2 \pi-\frac{4 m \pi}{j+1}\right)=-\sin \left(\frac{4 m \pi}{j+1}\right) .
\end{aligned}
$$

From the above discussion, we deduce that

$$
\int_{K_{m}} \sin ^{2} x \mathrm{~d} x=\int_{K_{m}^{\prime}} \sin ^{2} x \mathrm{~d} x .
$$

Similarly, we can have

$$
\int_{I_{m}} \sin ^{2} x \mathrm{~d} x=\int_{I_{m}^{\prime}} \sin ^{2} x \mathrm{~d} x
$$

3.2. $j \equiv 0(\bmod 4)$. Assume $j \equiv 0(\bmod 4)$. We see that

$$
\begin{aligned}
& \cos \theta_{p} \sin \left((j+1) \theta_{p}\right)>0 \Longleftrightarrow \theta_{p} \in \bigcup_{m=1}^{(j / 4)}\left(\frac{(2 m-2) \pi}{j+1}, \frac{(2 m-1) \pi}{j+1}\right) \cup\left(\frac{j \pi}{2(j+1)}, \frac{\pi}{2}\right) \bigcup_{m=(j / 4)+1}^{(j / 2)}\left(\frac{(2 m-1) \pi}{j+1}, \frac{2 m \pi}{j+1}\right), \\
& \cos \theta_{p} \sin \left((j+1) \theta_{p}\right)<0 \Longleftrightarrow \theta_{p} \in \bigcup_{m=1}^{(j / 4)}\left(\frac{(2 m-1) \pi}{j+1}, \frac{2 m \pi}{j+1}\right) \cup\left(\frac{\pi}{2}, \frac{(j+2) \pi}{2(j+1)}\right) \bigcup_{m=(j / 4)+1}^{(j / 2)}\left(\frac{2 m \pi}{j+1}, \frac{(2 m+1) \pi}{j+1}\right) .
\end{aligned}
$$

Obviously,

$$
\begin{aligned}
& \bigcup_{m=(j / 4)+1}^{(j / 2)}\left(\frac{(2 m-1) \pi}{j+1}, \frac{2 m \pi}{j+1}\right)=\bigcup_{m=1}^{(j / 4)}\left(\frac{(j-2 m+1) \pi}{j+1}, \frac{(j-2 m+2) \pi}{j+1}\right), \\
& \bigcup_{m=(j / 4)+1}^{(j / 2)}\left(\frac{2 m \pi}{j+1}, \frac{(2 m+1) \pi}{j+1}\right)=\bigcup_{m=1}^{(j / 4)}\left(\frac{(j-2 m+2) \pi}{j+1}, \frac{(j-2 m+3) \pi}{j+1}\right) .
\end{aligned}
$$


Next, we define the right-hand side of (44) and (45) as

$$
\begin{aligned}
C & =\bigcup_{m=1}^{(j / 4)}\left(\frac{(2 m-2) \pi}{j+1}, \frac{(2 m-1) \pi}{j+1}\right) \cup\left(\frac{j \pi}{2(j+1)}, \frac{\pi}{2}\right) \bigcup_{m=1}^{(j / 4)}\left(\frac{(j-2 m+1) \pi}{j+1}, \frac{(j-2 m+2) \pi}{j+1}\right) \\
& =\bigcup_{m=1}^{(j / 4)} I_{m} \cup J^{\prime} \bigcup_{m=1}^{(j / 4)} K_{m}, \\
D & =\bigcup_{m=1}^{(j / 4)}\left(\frac{(2 m-1) \pi}{j+1}, \frac{2 m \pi}{j+1}\right) \cup\left(\frac{\pi}{2}, \frac{(j+2) \pi}{2(j+1)}\right) \bigcup_{m=1}^{(j / 4)}\left(\frac{(j-2 m+2) \pi}{j+1}, \frac{(j-2 m+3) \pi}{j+1}\right) \\
& =\bigcup_{m=1}^{(j / 4)} K_{m}^{\prime} \cup J \bigcup_{m=1}^{(j / 4)} I_{m}^{\prime} .
\end{aligned}
$$

The rest of the proof runs as before.

\section{Proof of Theorem 2}

The next two lemmas are generalizations of Lemmas 1 and 2 and Lemmas 3.1 and 3.2 of Lao [17], respectively.

Lemma 5. Let $f, g \in H_{k}^{*}$ be two distinct cusp forms. Then, for $j \geq 1$ and $0 \leq i \leq j$, we have

$$
\begin{aligned}
& \sum_{p} \frac{\left(\lambda_{f}\left(p^{i}\right) \lambda_{f}\left(p^{j}\right)-\lambda_{g}\left(p^{i}\right) \lambda_{g}\left(p^{j}\right)\right)^{2}}{p^{s}} \\
& \quad=2\left(1+i-\delta_{1}\right) \sum_{p} \frac{1}{p^{s}}+O(1), \quad \text { as } s \longrightarrow 1^{+},
\end{aligned}
$$

where $\delta_{1}$ is defined as in (4).

Proof. By observing (11) and (13), we find that for $0 \leq i \leq j$,

$$
\begin{aligned}
\lambda_{f}\left(p^{i}\right) \lambda_{f}\left(p^{j}\right) & =\sum_{m=0}^{i} \sum_{m^{\prime}=0}^{j} \alpha_{f}(p)^{i-2 m} \alpha_{f}(p)^{j-2 m^{\prime}} \\
& =\sum_{l_{1}=0}^{i} \lambda_{\operatorname{sym}^{i+j-2 l_{1} f}}(p) .
\end{aligned}
$$

In particular, for $i=j$,

$$
\lambda_{f}^{2}\left(p^{j}\right)=\lambda_{\operatorname{sym}^{j} f \times \mathrm{sym}^{j} f}(p)=1+\sum_{l_{1}=1}^{j} \lambda_{\operatorname{sym}^{2 l_{1}} f}(p) .
$$

Hence, we have

$$
\lambda_{f}\left(p^{i}\right) \lambda_{f}\left(p^{j}\right) \times \lambda_{g}\left(p^{i}\right) \lambda_{g}\left(p^{j}\right)=\sum_{l_{1}=0}^{i} \sum_{l_{2}=0}^{i} \lambda_{\text {sym }^{i+j-2 l_{1}} f \times \text { sym }^{i+j-2 l_{2} g}}(p),
$$

$$
\begin{aligned}
\lambda_{f}^{2}\left(p^{i}\right) \lambda_{g}^{2}\left(p^{j}\right)= & +\sum_{l_{1}=1}^{i} \lambda_{\text {sym }^{2 l_{1}} f}(p)+\sum_{l_{2}=1}^{j} \lambda_{\text {sym }^{2 l_{2}} g}(p) \\
& +\sum_{l_{1}=1}^{i} \sum_{l_{2}=1}^{j} \lambda_{\text {sym }^{2 l_{1}} f \times \operatorname{sym}^{2 l_{2}} g}(p) .
\end{aligned}
$$

By (51) and (52), we see that for $j \geq 1$ and $0 \leq i \leq j$,

$$
\begin{aligned}
& \sum_{p} \frac{\left(\lambda_{f}\left(p^{i}\right) \lambda_{f}\left(p^{j}\right)-\lambda_{g}\left(p^{i}\right) \lambda_{g}\left(p^{j}\right)\right)^{2}}{p^{s}}=\sum_{p} \sum_{l_{1}=1}^{i} \frac{\lambda_{\mathrm{sym}^{2 l_{1} f}}(p)}{p^{s}}+\sum_{p} \sum_{l_{2}=1}^{j} \frac{\lambda_{\mathrm{sym}^{2 l_{2} f}}(p)}{p^{s}}+\sum_{p} \sum_{l_{1}=1}^{i} \frac{\lambda_{\mathrm{sym}^{2 l_{1} g}}(p)}{p^{s}} \\
& +\sum_{p} \sum_{l_{2}=1}^{j} \frac{\lambda_{\mathrm{sym}^{2 l_{2}} g}(p)}{p^{s}}+2 \sum_{p} \frac{1}{p^{s}}+\sum_{p} \sum_{l_{1}=1}^{i} \sum_{l_{2}=1}^{j} \frac{\lambda_{\mathrm{sym}^{2 l_{1}} f \times \mathrm{sym}^{2 l_{2}} f}(p)}{p^{s}} \\
& +\sum_{p} \sum_{l_{1}=1}^{i} \sum_{l_{2}=1}^{j} \frac{\lambda_{\mathrm{sym}^{2 l_{1}}} g \times \mathrm{sym}^{2 l_{2} g}(p)}{p^{s}}-2 \sum_{p} \sum_{l_{1}=0}^{i} \sum_{l_{2}=0}^{i} \frac{\lambda_{\mathrm{sym}^{i+j-2 l_{1}} f \times \mathrm{sym}^{i+j-2 l_{2} g}}(p)}{p^{s}} .
\end{aligned}
$$


Note that using (17), for $j \geq 1$ and $0 \leq i \leq j$, we have

$\sum_{p} \sum_{l_{1}=0}^{i} \sum_{l_{2}=0}^{i} \frac{\lambda_{s y m^{i+j-2 l_{1}} f \times \mathrm{sym}^{i+j-2 l_{2} g}}(p)}{p^{s}}=\delta_{1} \sum_{p} \frac{1}{p^{s}}+O(1), \quad$ as $s \longrightarrow 1^{+}$,

where $\delta_{1}$ is defined as in (4). In particular, taking $i=j=l_{1}=l_{2}$, we apply (2) and get

$$
\lambda_{\text {sym }^{i+j-2 l_{1}} f \times \text { sym }^{i+j-2 l_{2}} g}(p)=\lambda_{f}\left(p^{i+j-2 l_{1}}\right) \lambda_{g}\left(p^{i+j-2 l_{2}}\right)=1 .
$$

Now, using (15)-(17) and (54) to estimate the terms in (53), we obtain Lemma 5 immediately.
Lemma 6. Let $f, g \in H_{k}^{*}$ be two distinct cusp forms. Then, for $j \geq 1$ and $0 \leq i \leq j$, we have

$$
\left|\lambda_{f}\left(p^{i}\right) \lambda_{f}\left(p^{j}\right)-\lambda_{g}\left(p^{i}\right) \lambda_{g}\left(p^{j}\right)\right| \leq 2\left(i j+j+\delta_{2}\right),
$$

where $\delta_{2}$ is defined as in (5).

Proof. By (49), we know that for $0 \leq i \leq j$,

$$
\begin{aligned}
& \left|\lambda_{f}\left(p^{i}\right) \lambda_{f}\left(p^{j}\right)-\lambda_{g}\left(p^{i}\right) \lambda_{g}\left(p^{j}\right)\right| \\
& \quad=\sum_{l_{1}=0}^{i}\left|\lambda_{\text {sym }^{i+j-2 l_{1} f}}(p)-\lambda_{\text {sym }^{i+j-2 l_{1} g}}(p)\right| .
\end{aligned}
$$

Case 1. If $i+j$ is odd, then so is $i+j-2 l_{1}$. Then, (11) gives

$$
\begin{aligned}
& \lambda_{\text {sym }^{i+j-2 l_{1}} f}(p)=\alpha_{f}(p)^{i+j-2 l_{1}}+\alpha_{f}(p)^{i+j-2 l_{1}-2}+\cdots+\alpha_{f}(p)^{-i-j+2 l_{1}+2}+\alpha_{f}(p)^{-i-j+2 l_{1}}, \\
& \lambda_{\text {sym }^{i+j-2 l_{1}} g}(p)=\alpha_{g}(p)^{i+j-2 l_{1}}+\alpha_{g}(p)^{i+j-2 l_{1}-2}+\cdots+\alpha_{g}(p)^{-i-j+2 l_{1}+2}+\alpha_{g}(p)^{-i-j+2 l_{1}}
\end{aligned}
$$

Since $\left|\alpha_{f}(p)\right|=1$ (see (9)), we have

$$
\left|\lambda_{f}\left(p^{i}\right) \lambda_{f}\left(p^{j}\right)-\lambda_{g}\left(p^{i}\right) \lambda_{g}\left(p^{j}\right)\right| \leq 2(i j+j+i+1) .
$$

Case 2. If $i+j$ is even, then so is $i+j-2 l_{1}$. Similarly, we have

$$
\begin{aligned}
& \lambda_{\text {sym }^{i+j-2 l_{1}} f}(p)=\alpha_{f}(p)^{i+j-2 l_{1}}+\alpha_{f}(p)^{i+j-2 l_{1}-2}+\cdots+1+\cdots+\alpha_{f}(p)^{-i-j+2 l_{1}+2}+\alpha_{f}(p)^{-i-j+2 l_{1}}, \\
& \lambda_{\text {sym }^{i+j-2 l_{1}} g}(p)=\alpha_{g}(p)^{i+j-2 l_{1}}+\alpha_{g}(p)^{i+j-2 l_{1}-2}+\cdots+1+\cdots+\alpha_{g}(p)^{-i-j+2 l_{1}+2}+\alpha_{g}(p)^{-i-j+2 l_{1}}
\end{aligned}
$$

Thus,

$$
\left|\lambda_{f}\left(p^{i}\right) \lambda_{f}\left(p^{j}\right)-\lambda_{g}\left(p^{i}\right) \lambda_{g}\left(p^{j}\right)\right| \leq 2(i j+j) .
$$

By the above estimates, we conclude that for $j \geq 1$ and $0 \leq i \leq j$,

$$
\left|\lambda_{f}\left(p^{i}\right) \lambda_{f}\left(p^{j}\right)-\lambda_{g}\left(p^{i}\right) \lambda_{g}\left(p^{j}\right)\right| \leq 2\left(i j+j+\delta_{2}\right),
$$

where $\delta_{2}$ is defined as in (5).

Now, we are ready to prove Theorem 2 .

Proof. of Theorem 2. Let $f, g \in H_{k}^{*}$ be two distinct cusp forms. For $j \geq 1$ and $0 \leq i \leq j$, define

$$
h_{p}=\lambda_{f}\left(p^{i}\right) \lambda_{f}\left(p^{j}\right)-\lambda_{g}\left(p^{i}\right) \lambda_{g}\left(p^{j}\right) .
$$

We infer from (15) and (49) that

$$
\sum_{p} \frac{\lambda_{f}\left(p^{i}\right) \lambda_{f}\left(p^{j}\right)-\lambda_{g}\left(p^{i}\right) \lambda_{g}\left(p^{j}\right)}{p^{s}}=O(1), \quad \text { as } s \longrightarrow 1^{+} .
$$

Observe that $M=0$.

Moreover, we know from Lemmas 5 and 6 that $m=$ $2\left(1+i-\delta_{1}\right)$ and $B=2\left(i j+j+\delta_{2}\right)$.

Therefore, from the proof of Lemma 4, we have

$$
\frac{1+i-\delta_{1}}{4\left(i j+j+\delta_{2}\right)^{2}} \sum_{p} \frac{1}{p^{s}}+O(1) \leq \sum_{p \in \mathscr{A}} \frac{1}{p^{s}}, \quad \text { as } s \longrightarrow 1^{+},
$$

i.e., the set $\left\{p \mid \lambda_{f}\left(p^{i}\right) \lambda_{f}\left(p^{j}\right)<\lambda_{g}\left(p^{i}\right) \lambda_{g}\left(p^{j}\right)\right\}$ has analytic density at least $\left(\left(1+i-\delta_{1}\right) / 4\left(i j+j+\delta_{2}\right)^{2}\right)$.

\section{Proof of Theorem 3}

The following lemmas play an important role in the Proof of Theorem 3.

Lemma 7. Let $f, g \in H_{k}^{*}$ be two distinct cusp forms. Let $a, b, c_{1}, c_{2} \in \mathbb{R}$ with $a<b$. Then, for $j \geq 1$ and $s \longrightarrow 1^{+}$, we have 


$$
\begin{aligned}
& \sum_{p} \frac{\left(c_{1} \lambda_{f}\left(p^{j}\right)+c_{2} \lambda_{g}\left(p^{j}\right)-a\right)^{2}\left(c_{1} \lambda_{f}\left(p^{j}\right)+c_{2} \lambda_{g}\left(p^{j}\right)-b\right)^{2}}{p^{s}} \\
& =\left((j+1)\left(c_{1}^{4}+c_{2}^{4}\right)+\delta_{3}\left(c_{1}^{3}+c_{2}^{3}\right)+6 c_{1}^{2} c_{2}^{2}+\left((a+b)^{2}+2 a b\right)\left(c_{1}^{2}+c_{2}^{2}\right)+a^{2} b^{2}\right) \sum_{p} \frac{1}{p^{s}}+O(1),
\end{aligned}
$$

where $\delta_{3}$ is given in (7).

Proof. It is easy to verify that the sum on the left side of (66) equals

$$
\begin{aligned}
& -2 a b(a+b) \sum_{p} \frac{c_{1} \lambda_{f}\left(p^{j}\right)+c_{2} \lambda_{g}\left(p^{j}\right)}{p^{s}} \\
& +\left((a+b)^{2}+2 a b\right) \sum_{p} \frac{\left(c_{1} \lambda_{f}\left(p^{j}\right)+c_{2} \lambda_{g}\left(p^{j}\right)\right)^{2}}{p^{s}} \\
& -2(a+b) \sum_{p} \frac{\left(c_{1} \lambda_{f}\left(p^{j}\right)+c_{2} \lambda_{g}\left(p^{j}\right)\right)^{3}}{p^{s}} \\
& +\sum_{p} \frac{\left(c_{1} \lambda_{f}\left(p^{j}\right)+c_{2} \lambda_{g}\left(p^{j}\right)\right)^{4}}{p^{s}}+a^{2} b^{2} \sum_{p} \frac{1}{p^{s}}
\end{aligned}
$$

For the first sum in (67), by using (15), we get

$$
\sum_{p} \frac{c_{1} \lambda_{f}\left(p^{j}\right)+c_{2} \lambda_{g}\left(p^{j}\right)}{p^{s}}=O(1), \quad \text { as } s \longrightarrow 1^{+} .
$$

For the second sum in (67), one can easily obtain from (15), (17), and (50) that

$$
\begin{aligned}
& \sum_{p} \frac{\left(c_{1} \lambda_{f}\left(p^{j}\right)+c_{2} \lambda_{g}\left(p^{j}\right)\right)^{2}}{p^{s}} \\
& \quad=\left(c_{1}^{2}+c_{2}^{2}\right) \sum_{p} \frac{1}{p^{s}}+O(1), \quad \text { as } s \longrightarrow 1^{+} .
\end{aligned}
$$

As for the third sum in (67), we note that

$$
\begin{aligned}
\left(c_{1} \lambda_{f}\left(p^{j}\right)+c_{2} \lambda_{g}\left(p^{j}\right)\right)^{3}= & c_{1}^{3} \lambda_{f}^{3}\left(p^{j}\right)+3 c_{1}^{2} c_{2} \lambda_{f}^{2}\left(p^{j}\right) \lambda_{g}\left(p^{j}\right) \\
& +3 c_{1} c_{2}^{2} \lambda_{f}\left(p^{j}\right) \lambda_{g}^{2}\left(p^{j}\right)+c_{2}^{3} \lambda_{g}^{3}\left(p^{j}\right) .
\end{aligned}
$$

$$
\lambda_{f}^{2}\left(p^{j}\right) \lambda_{g}\left(p^{j}\right)=\lambda_{\text {sym }^{j} g}(p)+\sum_{l_{1}=1}^{j} \lambda_{\text {sym }^{2 l_{1}} f \times \text { sym }^{j} g}(p) .
$$

Noticing (11), (13), and (49), we clearly deduce that

$$
\begin{aligned}
\lambda_{f}^{3}\left(p^{j}\right)= & \lambda_{\text {sym }^{j} f}(p)+\sum_{l_{1}=1}^{j} \lambda_{\text {sym }^{j} f \times \text { sym }^{2 l_{1}} f}(p) \\
= & (j+1) \lambda_{\text {sym }^{j} f}(p)+\sum_{i=0}^{j-1}(i+1) \lambda_{\text {sym }^{3 j-2 i} f}(p) \\
& +\sum_{i=1}^{[j / 2]}(j-2 i+1) \lambda_{\text {sym }^{j-2 i} f}(p) .
\end{aligned}
$$

If $j$ is even and $j=2 i$, we apply (2) and get

$$
\lambda_{\text {sym-2if }}(p)=\lambda_{f}\left(p^{j-2 i}\right)=1 .
$$

Hence, we deduce from (15) and (17) that as $s \longrightarrow 1^{+}$,

$$
\sum_{p} \frac{\left(c_{1} \lambda_{f}\left(p^{j}\right)+c_{2} \lambda_{g}\left(p^{j}\right)\right)^{3}}{p^{s}}=\delta_{3}\left(c_{1}^{3}+c_{2}^{3}\right) \sum_{p} \frac{1}{p^{s}}+O(1),
$$

where $\delta_{3}$ is given in (7).

We now calculate the fourth sum in (67). By (50), we have

$$
\lambda_{f}^{4}\left(p^{j}\right)=1+2 \sum_{l_{1}=1}^{j} \lambda_{\text {sym }^{2 l_{1}} f}(p)+\sum_{l_{1}=1}^{j} \sum_{l_{2}=1}^{j} \lambda_{\text {sym }^{2 l_{1}} f \times \operatorname{sym}^{2 l_{2}} f}(p) .
$$

Combining the above result with (15) and (16) leads to

$$
\sum_{p} \frac{\lambda_{f}^{4}\left(p^{j}\right)}{p^{s}}=(j+1) \sum_{p} \frac{1}{p^{s}}+O(1), \quad \text { as } s \longrightarrow 1^{+} \text {. }
$$

From (15), (17), and (72), it is easy to check that as $s \longrightarrow 1^{+}$,

In view of (50), we have

$$
\begin{aligned}
\sum_{p} \frac{\lambda_{f}^{3}\left(p^{j}\right) \lambda_{g}\left(p^{j}\right)}{p^{s}}= & (j+1) \sum_{p} \frac{\lambda_{\text {sym }^{j} f \times \text { sym }^{j} g}(p)}{p^{s}}+\sum_{p} \sum_{i=0}^{j-1}(i+1) \frac{\lambda_{\text {sym }^{3 j-2 i} f \times \text { sym }^{j} g}(p)}{p^{s}} \\
& +\sum_{p} \sum_{i=1}^{[j / 2]}(j-2 i+1) \frac{\lambda_{\text {sym }^{j-2 i} f \times \text { sym }^{j} g}(p)}{p^{s}}=O(1) .
\end{aligned}
$$


Moreover, we infer from (15), (17), and (52) that $\sum_{p} \frac{\lambda_{f}^{2}\left(p^{j}\right) \lambda_{g}^{2}\left(p^{j}\right)}{p^{s}}=\sum_{p} \frac{1}{p^{s}}+O(1), \quad$ as $s \longrightarrow 1^{+}$.
By the above results, it is shown that as $s \longrightarrow 1^{+}$,

$$
\begin{aligned}
& \sum_{p} \frac{\left(c_{1} \lambda_{f}\left(p^{j}\right)+c_{2} \lambda_{g}\left(p^{j}\right)\right)^{4}}{p^{s}}=c_{1}^{4} \sum_{p} \frac{\lambda_{f}^{4}\left(p^{j}\right)}{p^{s}}+c_{2}^{4} \sum_{p} \frac{\lambda_{g}^{4}\left(p^{j}\right)}{p^{s}}+4 c_{1}^{3} c_{2} \sum_{p} \frac{\lambda_{f}^{3}\left(p^{j}\right) \lambda_{g}\left(p^{j}\right)}{p^{s}} \\
& +4 c_{1} c_{2}^{3} \sum_{p} \frac{\lambda_{f}\left(p^{j}\right) \lambda_{g}^{3}\left(p^{j}\right)}{p^{s}}+6 c_{1}^{2} c_{2}^{2} \sum_{p} \frac{\lambda_{f}^{2}\left(p^{j}\right) \lambda_{g}^{2}\left(p^{j}\right)}{p^{s}} \\
& =\left((j+1)\left(c_{1}^{4}+c_{2}^{4}\right)+6 c_{1}^{2} c_{2}^{2}\right) \sum_{p} \frac{1}{p^{s}}+O(1) . \\
& \left|\lambda_{f}\left(p^{j}\right)\right| \leq\left|\alpha_{f}(p)^{j}\right|+\left|\alpha_{f}(p)^{j-2}\right|+\cdots+\left|\alpha_{f}(p)^{-j+2}\right| \\
& +\left|\alpha_{f}(p)^{-j}\right| \leq(j+1) .
\end{aligned}
$$
into (67).

Lemma 8. Let $f, g \in H_{k}^{*}$ be two distinct cusp forms. Let $a, b, c_{1}, c_{2} \in \mathbb{R}$ with $a<b$. Then, for $j \geq 1$, we have

$$
\begin{aligned}
& \left|\left(c_{1} \lambda_{f}\left(p^{j}\right)+c_{2} \lambda_{g}\left(p^{j}\right)-a\right)\left(c_{1} \lambda_{f}\left(p^{j}\right)+c_{2} \lambda_{g}\left(p^{j}\right)-b\right)\right| \\
& \leq(j+1)^{2}\left(\left|c_{1}\right|+\left|c_{2}\right|\right)^{2}+(j+1)(|a|+|b|)\left(\left|c_{1}\right|+\left|c_{2}\right|\right)+|a b| .
\end{aligned}
$$

By similar computations, we infer from (13) and (50) that

$$
\begin{aligned}
\left|\lambda_{f}\left(p^{j}\right) \lambda_{g}\left(p^{j}\right)\right| & \leq(j+1)^{2}, \\
\left|\lambda_{f}^{2}\left(p^{j}\right)\right| & \leq(j+1)^{2} .
\end{aligned}
$$

For $j \geq 1$, we have

Proof. It follows from (9) and (11) that

$$
\begin{aligned}
& \left(c_{1} \lambda_{f}\left(p^{j}\right)+c_{2} \lambda_{g}\left(p^{j}\right)-a\right)\left(c_{1} \lambda_{f}\left(p^{j}\right)+c_{2} \lambda_{g}\left(p^{j}\right)-b\right) \\
& =c_{1}^{2} \lambda_{f}^{2}\left(p^{j}\right)+c_{2}^{2} \lambda_{g}^{2}\left(p^{j}\right)+2 c_{1} c_{2} \lambda_{f}\left(p^{j}\right) \lambda_{g}\left(p^{j}\right)-(a+b)\left(c_{1} \lambda_{f}\left(p^{j}\right)+c_{2} \lambda_{g}\left(p^{j}\right)\right)+a b .
\end{aligned}
$$

According to the triangle inequality, we draw the conclusion.

Proof. of Theorem 3. Let $f, g \in H_{k}^{*}$ be two distinct cusp forms. It is easy to verify from (15), (17), (50), and (83) that

$$
\sum_{p} \frac{\left(c_{1} \lambda_{f}\left(p^{j}\right)+c_{2} \lambda_{g}\left(p^{j}\right)-a\right)\left(c_{1} \lambda_{f}\left(p^{j}\right)+c_{2} \lambda_{g}\left(p^{j}\right)-b\right)}{p^{s}}
$$

$$
=\left(c_{1}^{2}+c_{2}^{2}+a b\right) \sum_{p} \frac{1}{p^{s}}+O(1), \quad \text { as } s \longrightarrow 1^{+} .
$$


Combining the above result with Lemmas 7 and 8 , Theorem 3 follows apparently by applying Lemma 4 with

$$
\left\{\begin{array}{l}
h_{p}=\left(c_{1} \lambda_{f}\left(p^{j}\right)+c_{2} \lambda_{g}\left(p^{j}\right)-a\right)\left(c_{1} \lambda_{f}\left(p^{j}\right)+c_{2} \lambda_{g}\left(p^{j}\right)-b\right), \\
B=(j+1)^{2}\left(\left|c_{1}\right|+\left|c_{2}\right|\right)^{2}+(j+1)(|a|+|b|)\left(\left|c_{1}\right|+\left|c_{2}\right|\right)+|a b|, \\
m=(j+1)\left(c_{1}^{4}+c_{2}^{4}\right)+\delta_{3}\left(c_{1}^{3}+c_{2}^{3}\right)+6 c_{1}^{2} c_{2}^{2}+\left((a+b)^{2}+2 a b\right)\left(c_{1}^{2}+c_{2}^{2}\right)+a^{2} b^{2}, \\
M=c_{1}^{2}+c_{2}^{2}+a b .
\end{array}\right.
$$

\section{Data Availability}

The data used to support the findings of this study are included within the article.

\section{Conflicts of Interest}

The authors declare that they have no conflicts of interest.

\section{Acknowledgments}

This work was supported by the Natural Science Foundation of Shandong Province (Grant no. ZR2018MA003).

\section{References}

[1] G. Lü, "The sixth and eighth moments of fourier coefficients of cusp forms," Journal of Number Theory, vol. 129, no. 11, pp. 2790-2800, 2009.

[2] G. Lü, "On general divisor problems involving hecke eigenvalues," Acta Mathematica Hungarica, vol. 135, no. 1-2, pp. 148-159, 2012.

[3] G. Lü, "Shifted convolution sums of fourier coefficients with divisor functions," Acta Mathematica Hungarica, vol. 146, no. 1, pp. 86-97, 2015.

[4] H. F. Liu and R. Zhang, "Some problems involving hecke eigenvalues," Acta Mathematica Hungarica, vol. 159, no. 1, pp. 287-298, 2019.

[5] Y.-K. Lau and G. Lü, "Sums of fourier coefficients of cusp forms," The Quarterly Journal of Mathematics, vol. 62, no. 3, pp. 687-716, 2011.

[6] J. Wu and W. Zhai, "Distribution of hecke eigenvalues of newforms in short intervals," The Quarterly Journal of Mathematics, vol. 64, no. 2, pp. 619-644, 2013.

[7] D. Zhang, Y.-K. Lau, and Y. Wang, "Remark on the paper "on products of fourier coefficients of cusp forms"” Archiv der Mathematik, vol. 108, no. 3, pp. 263-269, 2017.

[8] P. Deligne, "La conjecture de Weil. I," Publications Mathématiques de l'IHÉS, vol. 43, no. 1, pp. 273-307, 1974.

[9] J. Meher, K. D. Shankhadhar, and G. K. Viswanadham, "On the coefficients of symmetric power $L$-functions," International Journal of Number Theory, vol. 14, no. 3, pp. 813-824, 2018.

[10] T. Barnet-Lamb, D. Geraghty, M. Harris, and R. Taylor, "A family of calabi-yau varieties and potential automorphy II," Publications of the Research Institute for Mathematical Sciences, vol. 47, pp. 29-98, 2011.

[11] M. A. Amri, "Simultaneous sign change and equidistribution of signs of fourier coefficients of two cusp forms," Archiv der Mathematik, vol. 111, no. 3, pp. 257-266, 2018.
[12] P.-J. Wong, "On the chebotarev-sato-tate phenomenon," Journal of Number Theory, vol. 196, pp. 272-290, 2019.

[13] E. Kowalski, Y.-K. Lau, K. Soundararajan, and J. Wu, "On modular signs," Mathematical Proceedings of the Cambridge Philosophical Society, vol. 149, no. 3, pp. 389-411, 2010.

[14] K. Matomäki, "On signs of fourier coefficients of cusp forms," Mathematical Proceedings of the Cambridge Philosophical Society, vol. 152, no. 2, pp. 207-222, 2012.

[15] L. Chiriac, "Comparing hecke eigenvalues of newforms," Archiv der Mathematik, vol. 109, no. 3, pp. 223-229, 2017.

[16] L. Chiriac, "On the number of dominating fourier coefficients of two newforms," Proceedings of the American Mathematical Society, vol. 146, no. 10, pp. 4221-4224, 2018.

[17] H. X. Lao, "On comparing hecke eigenvalues of cusp forms," Acta Mathematica Hungarica, vol. 160, no. 1, pp. 58-71, 2020.

[18] L. Chiriac and A. Jorza, "Comparing hecke coefficients of automorphic representations," Transactions of the American Mathematical Society, vol. 372, no. 12, pp. 8871-8896, 2019.

[19] J. Newton and J. A. Thorne, "Symmetric power functoriality for holomorphic modular forms," 2020, http://arxiv.org/abs/ 1912.11261v2.

[20] L. Dong, H. Liu, and D. Zhang, "Zero density estimates for automorphic L-functions of $G L_{m}$," Acta Mathematica Hungarica, vol. 148, no. 1, pp. 191-210, 2016.

[21] H. Iwaniec, Topics in Classical Automorphic Forms, American Mathematical Society, Providence, RI, USA, 1997.

[22] Y. Jiang, G. Lü, and X. Yan, "Mean value theorem connected with fourier coefficients of hecke-maass forms for $S L(m, \mathbb{Z})$," Mathematical Proceedings of the Cambridge Philosophical Society, vol. 161, no. 2, pp. 339-356, 2016.

[23] H.-F. Liu, "Mean value estimates of the coefficients of product L-functions," Acta Mathematica Hungarica, vol. 156, no. 1, pp. 102-111, 2018.

[24] H. Lao, M. McKee, and Y. Ye, "Asymptotics for cuspidal representations by functoriality from GL (2)," Journal of Number Theory, vol. 164, pp. 323-342, 2016.

[25] H. Lao and A. Sankaranarayanan, "The distribution of fourier coefficients of cusp forms over sparse sequences," Acta Arithmetica, vol. 163, no. 2, pp. 101-110, 2014.

[26] H. Lao and H. Wei, " $\Omega$-result on coefficients of automorphic $L$-functions over sparse sequences," Journal of the Korean Mathematical Society, vol. 52, no. 5, pp. 945-954, 2015.

[27] P. Song, W. Zhai, and D. Zhang, "Power moments of hecke eigenvalues for congruence group," Journal of Number Theory, vol. 198, pp. 139-158, 2019.

[28] D. Zhang and Y. Wang, "Higher-power moments of Fourier coefficients of holomorphic cusp forms for the congruence subgroup $\Gamma_{0}(N)$," The Ramanujan Journal, vol. 47, no. 3, pp. $685-700,2018$. 
[29] L. Clozel and J. A. Thorne, "Level-raising and symmetric power functoriality, I," Compositio Mathematica, vol. 150, no. 5, pp. 729-748, 2014.

[30] L. Clozel and J. A. Thorne, "Level-raising and symmetric power functoriality. II," Annals of Mathematics, vol. 181, no. 2, pp. 303-359, 2015.

[31] L. Clozel and J. A. Thorne, "Level-raising and symmetric power functoriality, III," Duke Mathematical Journal, vol. 166, pp. 325-402, 2017.

[32] L. Dieulefait, "Automorphy of $\operatorname{Symm}^{5}(G L(2))$ and base change," Journal de Mathématiques Pures et Appliquées, vol. 104, no. 4, pp. 619-656, 2015.

[33] S. Gelbart and H. Jacquet, "A relation between automorphic representations of GL (2) and GL (3)," Annales Scientifiques de l'Ecole Normale Supérieure, vol. 11, no. 4, pp. 471-542, 1978.

[34] H. H. Kim, "Functoriality for the exterior square of $G L_{4}$ and the symmetric fourth of $G L_{2}$. appendix 1 by D. Ramakrishnan and appendix 2 by H. H. Kim and P. Sarnak," Journal of the American Mathematical Society, vol. 16, pp. 139-183, 2003.

[35] H. H. Kim and F. Shahidi, "Functorial products for $G L_{2} \times G L_{3}$ and the symmetric cube for $G L_{2}$," The Annals of Mathematics, vol. 155 , no. 3, pp. 837-893, 2002.

[36] H. H. Kim and F. Shahidi, "Cuspidality of symmetric powers with applications," Duke Mathematical Journal, vol. 112, pp. 177-197, 2002.

[37] H. Jacquet and J. A. Shalika, "On euler products and the classification of automorphic representations I," American Journal of Mathematics, vol. 103, no. 3, pp. 499-558, 1981.

[38] H. Jacquet and J. A. Shalika, "On euler products and the classification of automorphic forms II," American Journal of Mathematics, vol. 103, no. 4, pp. 777-815, 1981.

[39] Y.-K. Lau and J. Wu, "A density theorem on automorphic $L$ functions and some applications," Transactions of the American Mathematical Society, vol. 358, pp. 441-472, 2006.

[40] Z. Rudnick and P. Sarnak, "Zeros of principal $L$-functions and random matrix theory," Duke Mathematical Journal, vol. 81, pp. 269-232, 1996.

[41] F. Shahidi, "On certain L-functions," American Journal of Mathematics, vol. 103, no. 2, pp. 297-355, 1981.

[42] F. Shahidi, "Third symmetric power $L$-functions for $G L(2), "$ Compositio Mathematica, vol. 70, pp. 245-273, 1989. 